\title{
Imperceptible Somatosensory Stimulation Alters Sensorimotor Background Rhythm and Connectivity
}

\author{
저니 Nierhaus, ${ }^{1,2,3}$ @Norman Forschack, ${ }^{2,4}$ @Sophie K. Piper, ${ }^{1}$ Susanne Holtze, ${ }^{1}$ Thomas Krause, ${ }^{1,5}$ Birol Taskin, ${ }^{1,5}$ \\ Xiangyu Long, ${ }^{2}$ Johannes Stelzer, ${ }^{2}$ Daniel S. Margulies, ${ }^{2,3}$ Jens Steinbrink, ${ }^{1,5}$ and Arno Villringer ${ }^{1,2,3,5}$ \\ ${ }^{1}$ Berlin Neuroimaging Center and Department of Neurology, Charité Universitätsmedizin Berlin, 10117 Berlin, Germany, ${ }^{2}$ Max-Planck-Institute for Human \\ Cognitive and Brain Sciences, Department of Neurology, 04303 Leipzig, Germany, ${ }^{3}$ Mind-Brain Institute and Berlin School of Mind and Brain, Charité and \\ Humboldt-University, 10117 Berlin, Germany, ${ }^{4}$ Department of Psychology, University of Leipzig, 04109 Leipzig, Germany, and ${ }^{5}$ Center for Stroke Research \\ Berlin, Charité Universitätsmedizin Berlin, 10117 Berlin, Germany
}

Most sensory input to our body is not consciously perceived. Nevertheless, it may reach the cortex and influence our behavior. In this study, we investigated noninvasive neural signatures of unconscious cortical stimulus processing to understand mechanisms, which (1) prevent low-intensity somatosensory stimuli from getting access to conscious experience and which (2) can explain the associated impediment of conscious perception for additional stimuli. Stimulation of digit 2 in humans far below the detection threshold elicited a cortical evoked potential (P1) at $60 \mathrm{~ms}$, but no further somatosensory evoked potential components. No event-related desynchronization was detected; rather, there was a transient synchronization in the alpha frequency range. Using the same stimulation during fMRI, a reduced centrality of contralateral primary somatosensory cortex (SI) was found, which appeared to be mainly driven by reduced functional connectivity to frontoparietal areas. We conclude that after subthreshold stimulation the (excitatory) feedforward sweep of bottom-up processing terminates in SI preventing access to conscious experience. We speculate that this interruption is due to a predominance of inhibitory processing in SI. The increase in alpha activity and the disconnection of SI from frontoparietal areas are likely correlates of an elevated perception threshold and may thus serve as a gating mechanism for the access to conscious experience.

Key words: EEG background rhythms; fMRI; functional connectivity; somatosensory evoked potentials; subthreshold electrical stimulation; unconscious

\section{Introduction}

The human body is permanently exposed to sensory input, most of it, however, escapes conscious perception. Our nervous system seems to automatically categorize sensory input as either "relevant" or "irrelevant" for conscious experience. Based on detection curves for sensory stimuli at different intensities that typically have a sigmoid shape, one can define three categories of stimuli: (1) above a certain intensity, stimuli are (almost) always consciously perceived (suprathreshold); (2) stimuli at lower intensity (near-threshold) are perceived only in a certain percentage of trials; and, finally, (3) below a certain ("absolute") detection threshold, stimuli are never perceived (more precisely: not more than null trials). Accordingly, we label these imperceptible stimuli as subthreshold. One might think that neural processing of such subthreshold stimuli should terminate very early

Received Sept. 11, 2014; revised Jan. 30, 2015; accepted Feb. 16, 2015.

Author contributions: T.N., J. Steinbrink, and A.V. designed research;T.N., N.F., S.P., S.H., T.K., and B.T. performed research; T.N. and D.M. contributed unpublished reagents/analytic tools; T.N., N.F., X.L., and J.Stelzer analyzed data; T.N., N.F., D.M., J. Steinbrink, and A.V. wrote the paper.

We thank Sylvia Stasch for technical support during EEG recordings as well as Annett Wiedemann and Sylvie Neubert for technical support during MRI measurements.

The authors declare no competing financial interests.

Correspondence should be addressed to Till Nierhaus, Berlin Neuroimaging Center and Department of Neurology, Charité Universitätsmedizin Berlin, Charitéplatz 1, 10117 Berlin, Germany. E-mail: till.nierhaus@charite.de.

DOI:10.1523/JNEUROSCI.3806-14.2015

Copyright $\odot 2015$ the authors $\quad 0270-6474 / 15 / 355917-09 \$ 15.00 / 0$ in the bottom-up chain of sensory processing. However, previous invasive recordings (Libet et al., 1967, Ray et al., 1999) showed at least one early SEP component, indicating that the information reaches the primary somatosensory cortex (SI).

Using analogous subthreshold stimulation as Libet et al. (1967), we have previously shown that trains of such stimuli induce a negative BOLD fMRI signal in contralateral SI, SII, and SMA, and impede stimulus processing in the somatosensory system (Blankenburg et al., 2003, Taskin et al., 2008). While this finding generally confirms the notion that subthreshold stimuli induce neural processing in the somatosensory cortex, it is still unknown which neural processes after subthreshold stimulation (1) prevent access to conscious experience and (2) underlie the impaired detection of (other) somatosensory stimuli.

To address these issues, we performed two series of experiments using EEG and fMRI, respectively, during subthreshold electrical stimulation of finger nerves. In both experiments, we compared effects of subthreshold stimulation to those elicited by suprathreshold stimulation. For our EEG experiments, we hypothesized (1) to find early SEP components corresponding to results of the previous invasive recordings (Libet et al., 1967, Ray et al., 1999), which so far have never been confirmed noninvasively. Given that later components correlate with conscious experience (Zhang and Ding, 2010, Auksztulewicz and Blankenburg, 2013), we expected an attenuation or lack of later components. 
Regarding another key feature of the EEG response to (suprathreshold) somatosensory stimulation, the desynchronization of background alpha $(\mathrm{mu})$ activity (event-related desynchronization, $E R D$ ), which is thought to reflect increased local neuronal processing, we suspected (2) that ERD would be attenuated or even absent after subthreshold stimulation.

Finally, given the lack of conscious experience and our previous findings that trains of subthreshold stimuli impede perception and locally decrease the BOLD signal in the sensorimotor system (Blankenburg et al., 2003), we hypothesized that (3) subthreshold stimulation may affect functional connectivity between somatosensory areas and regions involved in conscious perception. To test this hypothesis, the same stimulation paradigm as in the EEG experiment was applied during fMRI and compared with restingstate measurements in a functional connectivity analysis.

\section{Materials and Methods \\ Subjects}

In this study an EEG and an fMRI experiment were performed, both consisting of two sessions: 40 healthy volunteers participated in the first EEG session (age range 19-31 years, mean $25.3 \pm 2.7$ years SD; 21 females) and 41 healthy volunteers participated in the second EEG session (age range $20-33$ years, mean $25.8 \pm 2.9$ years SD; 18 females); 16 subjects were measured in the first fMRI session (age range 20-32 years, mean $25.6 \pm 3.3$ years SD; 8 females) and another 16 subjects were measured in the second fMRI session (age range 22-34 years, mean $27.0 \pm 3.3$ years SD; 9 females). All subjects were right-handed [laterality score according to the Oldfield questionnaire: $89.0 \pm 12.9$ (SD) over a range of -100 (fully left-handed) to 100 (fully right-handed); Oldfield, 1971]. All experiments were performed in accordance with the principles laid down in the declaration of Helsinki. Before participation, all volunteers underwent a comprehensive neurological examination and had no history of neurological or psychiatric diseases or were on any medication. Due to defective or artificial EEG recording, one subject had to be excluded in the first EEG session (resulting in 39 datasets), and four subjects had to be excluded in the second EEG session (resulting in 37 datasets).

\section{Experimental procedures}

\section{Electrical stimulation}

Electrical finger nerve stimulation was performed with a constantcurrent stimulator (DS7; Digitimer) using single square wave pulses with a duration of $200 \mu \mathrm{s}$, triggered with the stimulation software Presentation (Neurobehavioral Systems). In all experiments, steel wire ring electrodes were placed on the middle (anode) and the proximal (cathode) phalanx of the left index finger. For EEG session two, an additional pair of ring electrodes was placed on the right index finger. To ensure that subthreshold stimulations were always imperceptible, we determined the lowest intensity where subjects ever reported any sensation ("absolute perception threshold"). "Subthreshold" stimulation intensity was then set to $15 \%$ below this absolute perception threshold. In all experiments, subjects never reported any sensation due to the subthreshold stimulation intensity. Absolute perception thresholds were determined before and after each measurement block to control for potential drifts using the method of limits and a subsequent forced choice approach.

\section{EEG acquisition}

EEG session one. During stimulation blocks, EEG was recorded continuously from 32 scalp positions distributed over both hemispheres according to the international 10-20 system, using a commercial EEG acquisition system (BrainAmp; Brain Products). The mid-frontal electrode $(\mathrm{FPz})$ was used as reference and an electrode at the sternum served as ground. Skin electrode impedance was $<5 \mathrm{k} \Omega$ for all channels. EEG was recorded with a bandpass filter between $0.1 \mathrm{~Hz}$ and $1 \mathrm{kHz}$ and digitized with a sampling rate of $5 \mathrm{kHz}$.

To allow for proper detection of somatosensory evoked potentials (SEPs) and modulation of Rolandic rhythms, long interstimulus inter- vals were chosen. Subthreshold electrical finger nerve stimulation with an intensity $15 \%$ below absolute perception threshold was presented at a mean interstimulus interval of $4 \mathrm{~s}$ with a jitter of $\pm 500 \mathrm{~ms}$ during three measurement blocks of 15 min each, resulting in 675 trials per subject. For the control condition, a fourth block ( 3 min duration) with a suprathreshold stimulation intensity (clearly perceivable for the subject but at least $15 \%$ above absolute perception threshold) was appended, where stimuli were applied every $1 \pm 0.2 \mathrm{~s}$, resulting in 180 trials.

EEG session two. As a confirmatory experiment, a second EEG study was performed in a slightly modified setup with shorter blocks and with intermingled suprathreshold stimuli to prevent subjects getting tired ("by nothing happening"). Subthreshold stimulation at an intensity $15 \%$ below absolute perception threshold was presented at a mean interstimulus interval of $2 \mathrm{~s}$ with a jitter of $\pm 300 \mathrm{~ms}$ during eight measurement blocks of 2 min each, resulting in 480 trials per subject. Additionally, in each block up to four suprathreshold stimuli (clearly perceivable for the subject but at least 50\% above absolute perception threshold) were randomly presented to the left or the right hand. Due to the rare occurrence of the suprathreshold stimuli in this experiment we increased their intensity (50\% above threshold vs 15\% above threshold in EEG session one) to ensure their perception over the entire block. Subjects were instructed to relax and fixate a fixation cross.

An active electrode cap was used to record the EEG, sampling rate was $1 \mathrm{kHz}$; all other acquisition parameters were the same as in the first session.

\section{fMRI acquisition}

fMRI was measured using a 3 T Siemens Magnetom (Verio). BOLDsensitive images were acquired using a $\mathrm{T} 2^{*}$-weighted EPI sequence $\left(\mathrm{TR}=2000 \mathrm{~ms}, \mathrm{TE}=30 \mathrm{~ms}\right.$, flip angle $=90^{\circ}$, matrix $64 \times 64, \mathrm{FOV}=$ $19.2 \mathrm{~cm}$, in-plane resolution $=3 \mathrm{~mm} \times 3 \mathrm{~mm}$, slice thickness $=4 \mathrm{~mm}$, interslice gap $=1 \mathrm{~mm}, 30$ slices, interleaved). Measurement blocks of 180 volumes $(6 \mathrm{~min})$ were acquired. The first fMRI session consists of two blocks, one resting-baseline block followed by a second block during which subthreshold stimuli were applied every $4 \pm 0.5 \mathrm{~s}$ with intensity $15 \%$ below absolute perception threshold. In the second fMRI session, in addition to resting-baseline and subthreshold stimulation, we acquired an additional block with suprathreshold stimulation $(4 \pm 0.5 \mathrm{~s}$ with intensity $50 \%$ above absolute perception threshold, i.e., clearly perceivable for the subject). The order of subthreshold and suprathreshold stimulation blocks was counterbalanced across subjects.

\section{EEG data analysis}

EEG data analysis was performed off-line using custom-built MATLAB scripts (The MathWorks). After downsampling to $500 \mathrm{~Hz}$, the dataset of each subject (subthreshold and suprathreshold merged for EEG session one) underwent an independent component analysis (ICA) to remove sources of ocular artifacts ( $\mathrm{Li}$ et al., 2006). After back projection, data were subdivided into epochs and digitally filtered using a standard thirdorder bandpass Butterworth filter (low cutoff frequency $1 \mathrm{~Hz}$, high cutoff frequency $45 \mathrm{~Hz}$ ).

SEPs. Epochs were defined ranging from -600 to $1100 \mathrm{~ms}$ relative to stimulus onset. For the control condition in session one (suprathreshold), however, epochs of -200 to $600 \mathrm{~ms}$ were defined since mere suprathreshold stimulation was presented at a shorter interstimulus interval. Finally, data were downsampled to $250 \mathrm{~Hz}$ and SEPs were obtained by averaging all trials.

Statistical analysis was performed on electrode C4 (i.e., over the right somatosensory cortex contralateral to the stimulation site). The SEP was analyzed in a $400 \mathrm{~ms}$ epoch post stimulus. A baseline was defined as a 200 $\mathrm{ms}$ prestimulus epoch ( -200 to $0 \mathrm{~ms}$ ). Each sample point of the poststimulus epoch was successively compared with the mean prestimulus baseline value. For each comparison, a paired $t$ test $(p<0.05)$ was performed and the Bonferroni correction method was used to correct for multiple comparisons (100 tests, $\left.p_{B C}<0.0005\right)$.

Rolandic rhythms. Since Rolandic rhythms can be hidden under dominating occipital alpha activity, a preselection of "central" ICA components was performed before trial segmentation. For this purpose, all blocks were concatenated to run individual ICAs for EEG sessions one 
and two, respectively. Rolandic background rhythms are characterized by a central localization and two peaks in the power spectrum, at alpha $(8-15 \mathrm{~Hz})$ and beta $(16-30 \mathrm{~Hz})$ frequency bands, respectively, which desynchronize after suprathreshold stimulation. Based on this "operational definition of Rolandic rhythms," for each subject only those ICs were selected for which a central topography-the two respective peaks in the frequency spectrum and a desynchronization after suprathreshold stimulation-were identifiable. Using this procedure, 2-7 (mean $3 \pm 1$ $\mathrm{SD}$ ) independent components were selected per subject for EEG session one, and 1-4 (mean $2 \pm 1 \mathrm{SD}$ ) for EEG session two. The selected components were back projected and included in further analysis. After back projection of the "central" ICA components and segmentation according to subthreshold epochs defined above ( -600 to $1100 \mathrm{~ms}$ relative to stimulus onset), a wavelet analysis was performed for the frequencies from 4 to $30 \mathrm{~Hz}$ in $1 \mathrm{~Hz}$ increments to allow for a time-resolved frequency analysis of ERD. The wavelet transformation was performed on each single trial using a five-cycle long wavelet and subsequent averaging of the respective results. A $200 \mathrm{~ms}$ prestimulus epoch ( -200 to $0 \mathrm{~ms})$ was used to define a baseline.

Statistical analysis for EEG session one was performed on electrode C4 (same electrode as for the SEP analysis). For EEG session two, however, CP4 showed the main effect of ERD after the selection of "central" ICA components and thus was used for statistical analysis. Individual data were permuted by generating 5000 sets of pseudotrials and wavelet transformation was performed for each permutation. Permutations were pooled across subjects and the change to mean baseline activity was compared with the original set of trials.

\section{fMRI data analysis}

The general approach was a functional connectivity analysis based on "eigenvector centrality mapping" (ECM) as it has been performed previously for "resting-state" data (Lohmann et al., 2010). After determining ECMs for each condition, in a second step, these maps where compared between the different conditions ("no stimulation," "subthreshold stimulation," and "suprathreshold stimulation"). Since in two of the three conditions stimulations were applied, we considered removing direct stimulation-induced effects in the frequency or time domain. However, to use a stimulation-associated regressor (of no interest) in the time domain would have meant using such a regressor only for the two "stimulation conditions." To avoid this, we filtered out the main frequencies of the stimulation effect $(0.25 \mathrm{~Hz}$ and higher harmonics) by applying a bandpass filter $(0.01-0.2 \mathrm{~Hz})$. Thus, the same preprocessing was applied to all three conditions (no stimulation, subthreshold stimulation, and suprathreshold stimulation).

In more detail preprocessing of the fMRI data was performed using LIPSIA-toolbox (Lohmann et al., 2001): head motion correction, slice time correction, bandpass filtering $(0.01-0.2 \mathrm{~Hz})$, spatial smoothing (7 mm kernel), and normalization to the MNI305 space. Networks of correlated brain activity can be identified from the temporal correlations of an ROI time series of spontaneous BOLD fMRI signal with all other brain regions (Cordes et al., 2001; Gusnard and Raichle, 2001; Fox and Raichle, 2007; Margulies et al., 2010; Nierhaus et al., 2012). Several studies have shown that brain regions, which show correlated activity during a task, also appear correlated at rest, resulting in similar "maps" of connected brain areas involved in certain brain functions (Biswal et al., 1995; Cordes et al., 2000; Fox et al., 2005, 2007). Here, we used ECM (similar to Google's PageRank algorithm; Lohmann et al., 2010) as a data-driven analysis tool that characterizes each voxel's connectivity within the whole-brain network. The ECM algorithm calculates linear correlations for each voxel to every other voxel (correlation matrix A). This matrix is then rescaled to positive values. The eigenvector centrality of the $i$ th voxel is then defined as the ith entry of the (normalized) eigenvector of the largest eigenvalue of A. This procedure favors voxels that are strongly connected with many other voxels of high centrality. We computed a whole-brain ECM for each measurement block. The ECM results of session one (subthreshold stimulation compared with resting baseline) were then used to define an ROI for subsequent whole-brain, seed-based functional connectivity analysis. We used custom-built MATLAB scripts (The MathWorks) to calculate spatial correlation maps between the seed region and all other voxels in the brain. The head motion, averaged signal from CSF, and white matter were regressed out of fMRI data in the spatial correlation analysis.

It should be noted that the ECM analysis is a whole-brain approach without any prior assumption. Only in those areas, which showed a statistically significant alteration in "overall connectivity," did we subsequently perform a seed-based analysis. The question was as follows: Given that these areas show overall reduced connectivity compared with baseline, which are the most affected connections? In this step, no formal statistics was applied (double dipping); rather it was used to identify those areas that "contributed most" to the statistically significant effect of an overall reduced connectivity.

For the ECMs, voxelwise paired $t$ test comparisons over all subjects were used to calculate statistical significant differences between the different measurement blocks. For the spatial correlation maps resulting from seed-based connectivity analysis, a voxelwise paired $t$ test (contrasting the subthreshold stimulation block with the resting baseline of the first fMRI session) was used to calculate significant differences in functional connectivity. All resulting $T$-maps underwent a multiplecomparison procedure for significance thresholding based on Monte Carlo simulations with 1000 iterations (vmulticomp, LIPSIA toolbox; Lohmann et al., 2001). This method uses two features (cluster size and maximum $z$-value) to quantify a cluster as significant (Forman et al., 1995; Poline et al., 1997). We applied the cluster-size correction, using a voxelwise $z$-threshold of 2.57 and a significance level of $p<0.01$ on cluster level.

\section{Results}

Noninvasive detection of SEP following subthreshold stimulation

While previous invasive studies (Libet et al., 1967; Ray et al., 1999) strongly suggested the existence of at least one early component of an SEP at $\sim 50-60 \mathrm{~ms}$ following subthreshold stimulation, this component has not yet been demonstrated in noninvasive EEG recordings, probably due to its poor SNR. Therefore, in our EEG study we made sure that with a large number of trials and subjects, we would have enough SNR to identify even subtle evoked brain activity. Figure 1 (top) shows the grand average SEP following subthreshold stimulation (15\% below absolute perception threshold). An early positive component at $\sim 60 \mathrm{~ms}$ (P1, paired $t$ test mean baseline value vs 55-65 ms time interval; $p=0.0002$ ) can be clearly identified. This peak at $60 \mathrm{~ms}$ closely corresponds to the evoked activity measured invasively in previous studies (Libet et al., 1967; Ray et al., 1999). No later component was identified. The lower plot in Figure 1 shows the SEP following suprathreshold stimulation. The early positive component (P1) appears a bit earlier at $\sim 50 \mathrm{~ms}$ and clearly has a larger amplitude compared with the $\mathrm{P} 1$ elicited by subthreshold stimulation. In addition, a late negative component $\sim 170 \mathrm{~ms}$ (N1) is clearly seen.

The subthreshold and suprathreshold SEPs were lateralized to the right hemisphere, i.e., contralateral to the stimulation side (Fig. 2).

\section{Subthreshold stimulation increases pericentral rhythmic EEG activity}

Figure 3 shows the time-frequency plots for subthreshold $(A, B)$ and suprathreshold $(C)$ stimulation contralateral to the stimulation side. The wavelet analysis of the suprathreshold stimulation revealed the well studied effect of a stimulus-induced desynchronization in the alpha and beta range (Fig. $3 C$ ). For subthreshold stimulation, however, an increase of rhythmic activity was observed in both EEG studies. For the subthreshold stimulation in the first EEG session (Fig. 3A) a lower alpha-band activity (8-11 $\mathrm{Hz}$ ) with a maximum $\sim 80 \mathrm{~ms}$ post stimulus is followed by an 
upper alpha-band activity (12-15 Hz) with a maximum $\sim 200$ ms post stimulus. In EEG session two, subthreshold stimulation induced alpha-band activity $(8-12$ $\mathrm{Hz}$ ) from 50 to $450 \mathrm{~ms}$. For visualization, the results of the statistical permutation testing is shown in Figure 3, bottom. The duration and precise frequencies were somewhat different between the two EEG experiments, which may be related to the slightly different stimulation protocols and to effects such as spatial attention and/or vigilance. Indeed, in a follow-up study, we are currently providing evidence that spatial attention modulates the effect of subthreshold stimulation (Forschack et al., 2014). The effect of desynchronization for suprathreshold stimulation and the subthreshold stimulation-induced increase in Rolandic rhythmic activity were also lateralized to the right (contralateral) hemisphere (Fig. 2).

\section{Subthreshold stimulation reduces eigenvector centrality of SI}

ECM is a data-driven analysis tool based on BOLD signal changes in fMRI, which allows us to compare whole-brain functional connectivity at different states of the brain without prior spatial assumptions (Lohmann et al., 2010). In our fMRI study, we compared brain connectivity patterns for three different conditions: subthreshold stimulation versus suprathreshold stimulation versus no stimulation. In the first fMRI session we acquired a single resting-state (baseline) block and a single block with subthreshold stimulation in 16 subjects $(n=16)$. Thus EC maps with and without subthreshold stimulation can be compared with changes in whole-brain functional connectivity elicited by the continuous subthreshold input. The top row of maps in Figure 4 show the results of a paired $t$ test contrasting the eigenvector centrality of the subthreshold stimulation block versus the baseline block. We found reduced eigenvector centrality in the contralateral hand area of SI during subthreshold stimulation, i.e., the BOLD activity in this area is less connected to whole-brain network during subthreshold stimulation (Fig. 4, top). In the second fMRI session a block with suprathreshold stimulation was added to the resting baseline and subthreshold stimulation block and again 16 subjects were acquired $(n=16)$. Suprathreshold stimulation did not significantly modulate eigenvector centrality in SI, showing only small changes (left inferior frontal and right posterior temporal) in areas unrelated to primary somatosensory processing (Fig. 4, middle). A direct comparison of subthreshold and suprathreshold stimulation blocks (independent of the resting-state baseline), however, reconfirms our result of the first fMRI session, additionally showing little modulation in ipsilateral SI (Fig. 4, bottom).

\section{Subthreshold stimulation reduces functional connectivity from $S 1$ to frontoparietal areas}

While the comparison of EC maps at different states (subthreshold stimulation, suprathreshold stimulation, and no stimulation; Fig. 4) identify brain regions with altered overall connectivity during electrical stimulation, they do not show the specific brain areas to which connectivity has changed. Seed-based functional connectivity, however, provides information about increased or decreased connectivity of a predefined ROI ("seed") to all other brain regions. Since ECM analysis of subthreshold electrical stimulation resulted in reduced overall connectivity of contralateral hand area (Fig. 4, top), in a second step, we used this region as ROI for a seed-based functional connectivity analysis. Please note that — though we had a strong a priori hypothesis of contralateral SI to be affected by our stimulation protocol (Blankenburg et al., 2003) — we first used ECM as a completely data-driven analysis tool to obtain a "whole-brain connectivity map" without prior assumptions. Only based on the results of ECM we used contralateral SI as ROI for the seed-based functional connectivity analysis.

A paired $t$ test contrasting the resulting functional connectivity maps during the rest condition (no stimulation) with the subthreshold condition revealed the seed region to be significantly less correlated to medial parietal cortex (precuneus), posterior cingulate cortex, right inferior frontal gyrus, right operculum, left inferior parietal gyrus, and prefrontal cortex, as well as parts of cerebellum, during the subthreshold stimulation (Fig. 5).

\section{Discussion}

Using EEG and fMRI, we investigated neural signatures of unconscious cortical stimulus processing after subthreshold somatosensory stimulation. The goal was to identify mechanisms that (1) prevent access to conscious perception and (2) underlie behavioral consequences of subthreshold stimulation. The main findings are as follows. Subthreshold electrical single pulses generate an SEP peaking at $60 \mathrm{~ms}$ (P1), the amplitude of which is weaker than the P1 after suprathreshold stimulation and occurs $\sim 10$ ms later. No subsequent SEP components were detected. Subthreshold stimulation does not desynchronize $\mathrm{Mu}$-rhythm; rather, there is a transient increase between 50 and $400 \mathrm{~ms}$. Subthreshold stimulation decreases centrality of the contralateral SI hand area predominantly driven by reduced functional connectivity to frontoparietal areas.

Previous studies on the detection of near-threshold (Zhang and Ding, 2010, Auksztulewicz and Blankenburg, 2013) and masked suprathreshold (Schubert et al., 2006) stimuli found an unchanged P1 and attenuated (if present at all) N1 component for undetected compared with consciously perceived trials. We 


\section{subthreshold}

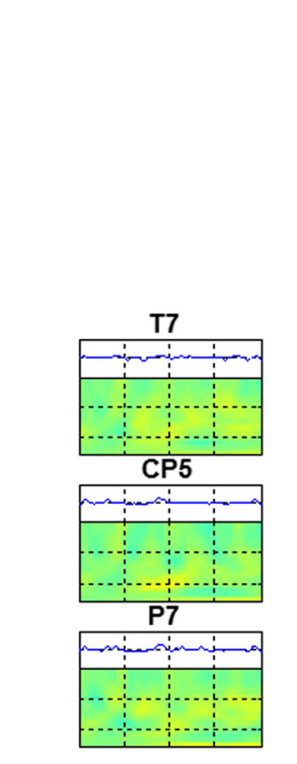

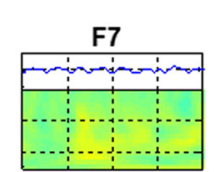

FC5

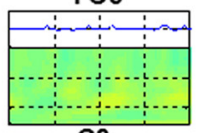

C3

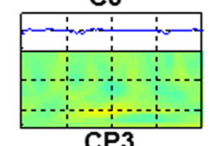

$\mathrm{CP} 3$

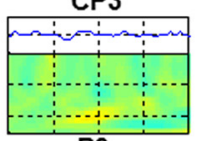

P3

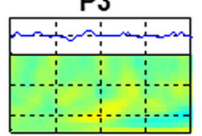

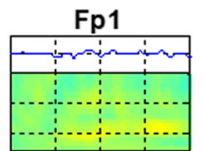

F3

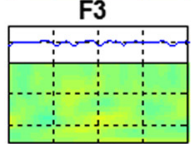

FC1
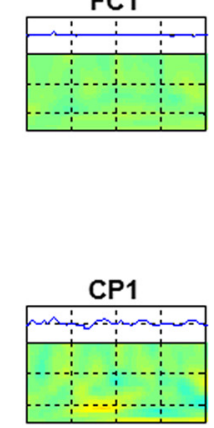
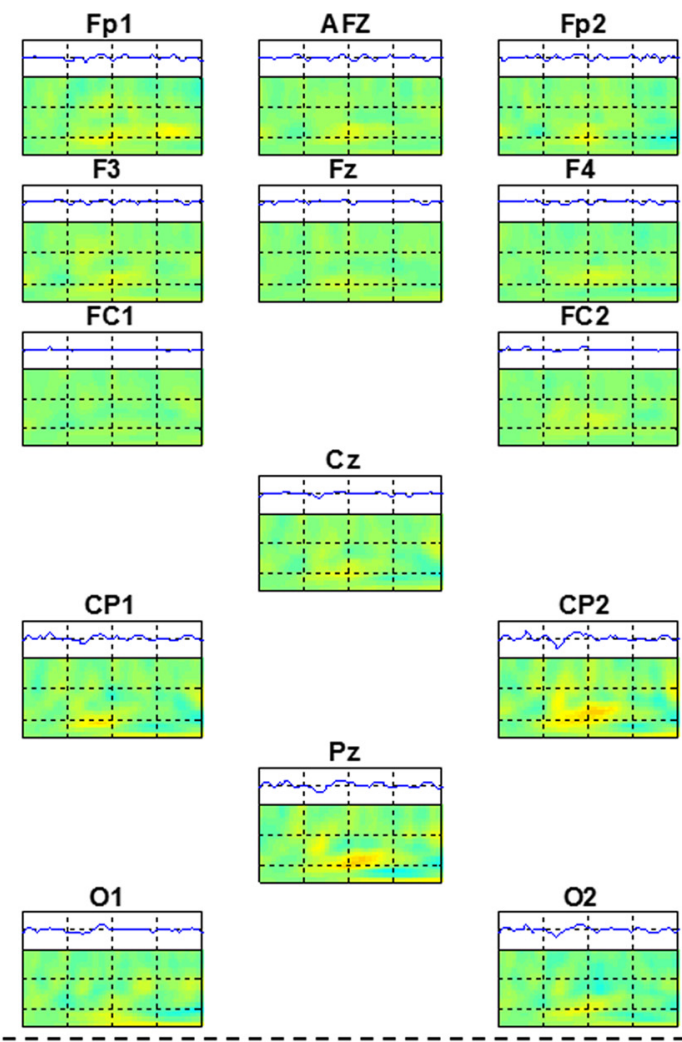

suprathreshold
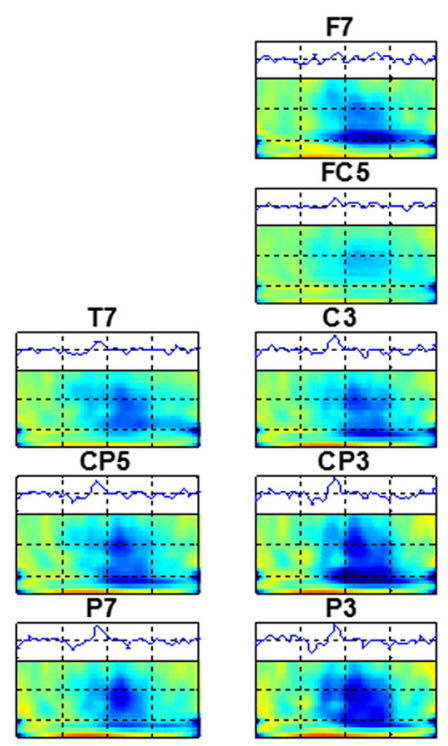

FC5

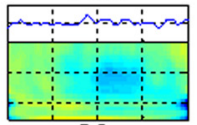

C3
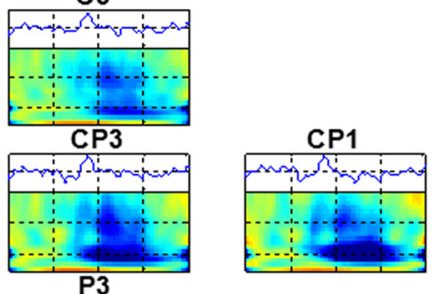

Fp1

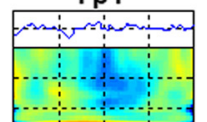

F3
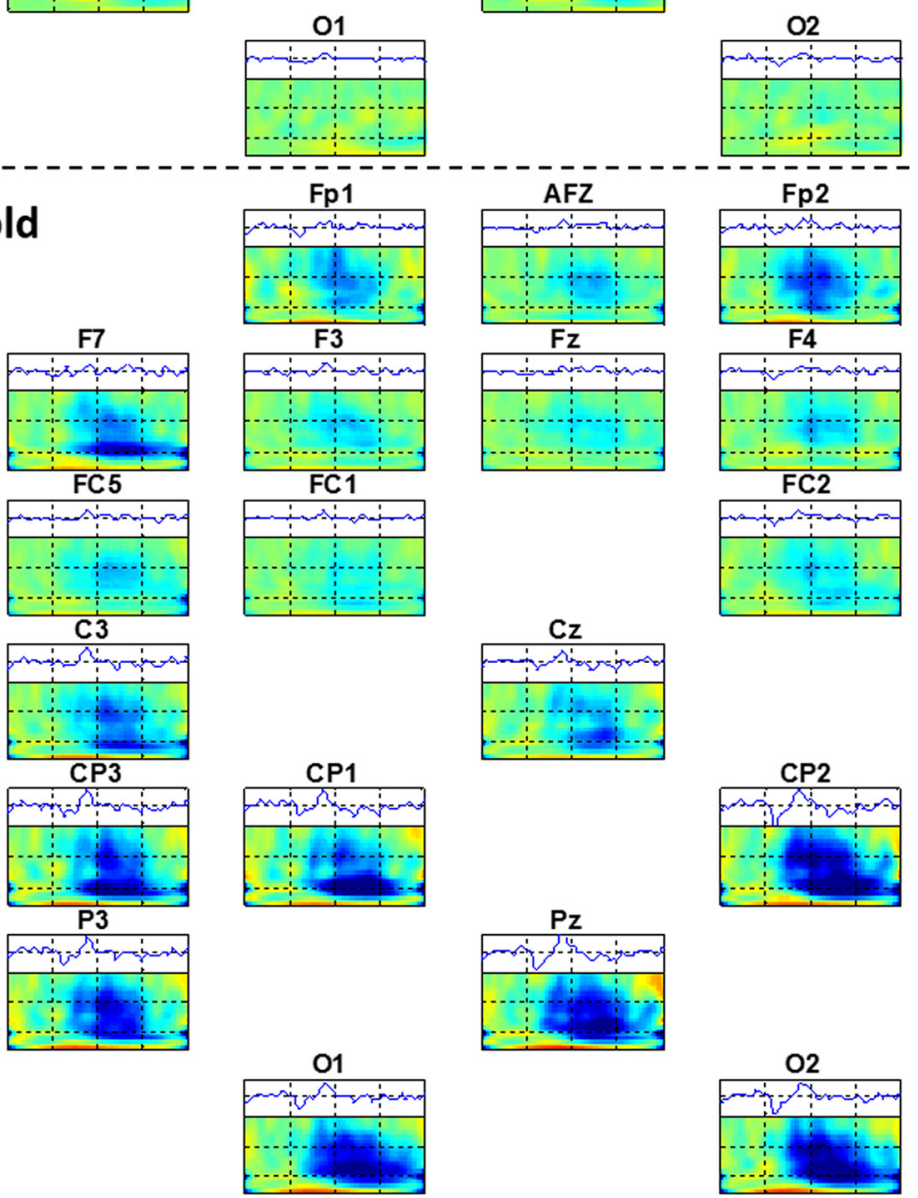

Figure 2. The oscillatory activity to subthreshold and suprathreshold stimulation is contralateralized. Grand average $(n=39)$ head plot of the SEP (blue curve) and the oscillatory activity (color-coded time-frequency plots) for subthreshold (top) and suprathreshold stimulation (bottom) in EEG session 1, after selection of central ICA components. Time is expressed relative to stimulation onset, and oscillatory activity is expressed relative to mean baseline power (baseline: $200 \mathrm{~ms}$ segment prestimulus).

extended these studies to (always imperceptible) subthreshold stimuli, for which we find a reduced and delayed P1 and absence of later components. Since our recordings show a similar shape and temporal kinetics as previous invasive studies (Libet et al.,
1967; Ray et al., 1999), we regard our findings as the first noninvasive demonstration of "subthreshold SEPs." P1 is a well known SEP component, which has been suggested to reflect an IPSP (Wikström et al., 1996), originating in BA1 within SI (Allison et 
al., 1992; Srisa-an et al., 1996). The relatively slow kinetics of the observed P1 following subthreshold stimulation is compatible with the temporal shape of cortical IPSPs, which are less sharp and have a smaller amplitude than EPSPs, due to substantially different synaptic driving forces (Whitehorn and Towe, 1968; Swadlow, 2003). However, given that excitatory and inhibitory processing in SI are always tightly coupled (Miller et al., 2001), it seems rather unlikely that at any given processing step there is just one type of synaptic activity (i.e., inhibitory) involved. Thus, a more appropriate view is that of a balance between excitatory and inhibitory activity (Isaacson and Scanziani, 2011). Given a lower stimulation threshold of inhibitory interneurons (as opposed to excitatory principal neurons), one can infer a preferential activation of inhibitory interneurons at low amplitude stimulation (Gil and Amitai, 1996; Swadlow, 2003). Thus, it seems plausible that the shift in peak timing as well as the lower amplitude of P1 reflect a modulation of the local excitation/inhibition ratio toward a dominance of inhibitory processing. No subsequent ERP component was detected suggesting that this predominant inhibition leads to a termination of the (mainly excitatory) bottom-up sweep at this time point. Specifically, there is no N1 component, which has been suggested to partly represent the neural correlates of conscious perception (Cauller, 1995), and to reflect activity in SI after feedback input from higher order cortices such as SII, PFC, and posterior parietal cortex (Cauller and Kulics, 1991; Knight et al., 1999; Staines et al., 2002; Golmayo et al., 2003). We think that subthreshold stimulation failed to trigger these feedback loops. Together, a plausible explanation of our results seems that the bottom-up sweep after subthreshold stimulation is terminated after $\sim 60 \mathrm{~ms}$ due to a predominance of feedforward inhibition in BAl preventing access to conscious experience.

The notion of predominant inhibition after subthreshold stimulation is further supported by alterations in Mu-rhythm and functional connectivity as outlined subsequently. After suprathreshold somatosensory stimulation, between 100 and $600 \mathrm{~ms}$, we confirm the well described ERD (Neuper and Pfurtscheller, 2001; Ploner et al., 2006; Stancák, 2006) in the alpha and beta frequency bands. An ERD is usually regarded as a sign of local ongoing stimulus processing. In contrast to this, for subthreshold stimulation, we find
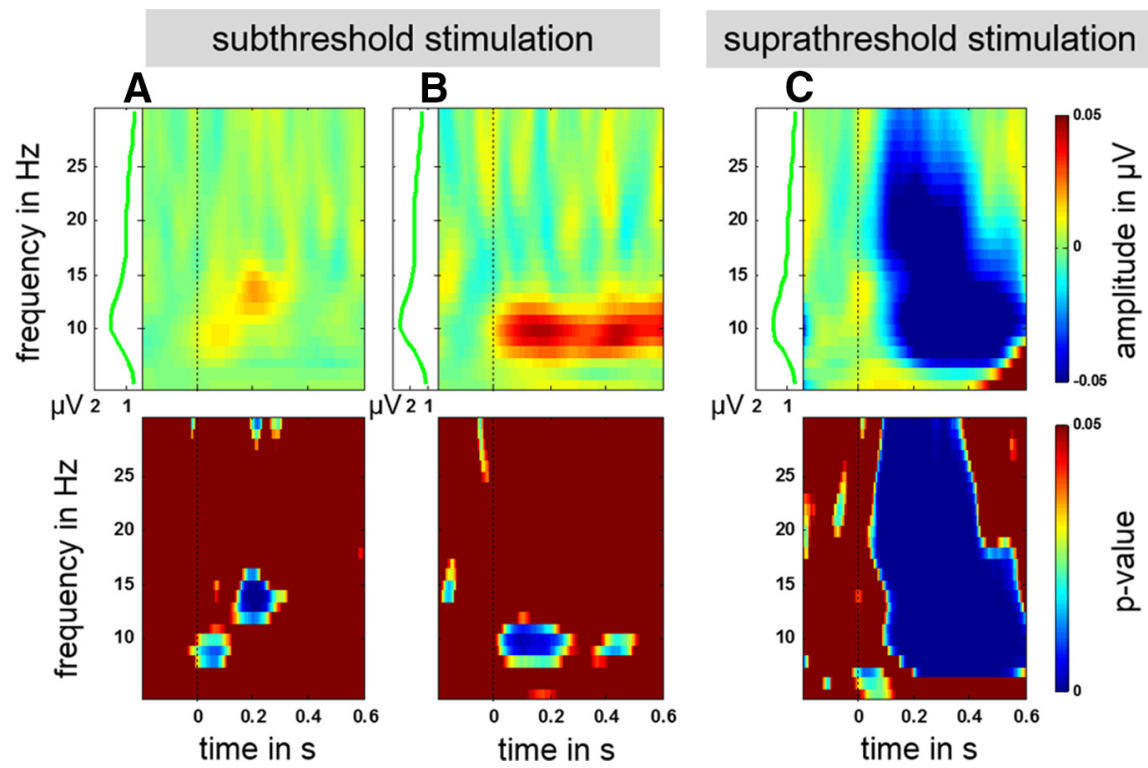

Figure 3. Subthreshold stimulation increases Rolandic rhythms. Grand average time-frequency plots (contralateral to stimulation side) for subthreshold ( $\boldsymbol{A}$, EEG session 1, electrode $(4, n=39 ; \boldsymbol{B}$, EEG session 2, electrode $(P 4, n=37)$ and suprathreshold ( $C$, EEG session 1, electrode $(4, n=39)$ stimulation after selection of central ICA components. Time is expressed relative to stimulation onset, and oscillatory activity is expressed relative to baseline power (baseline: $200 \mathrm{~ms}$ prestimulus). The green line on the left of the time-frequency plots indicates mean baseline power. Bottom plots indicate $p$ values derived from the permutation statistics.

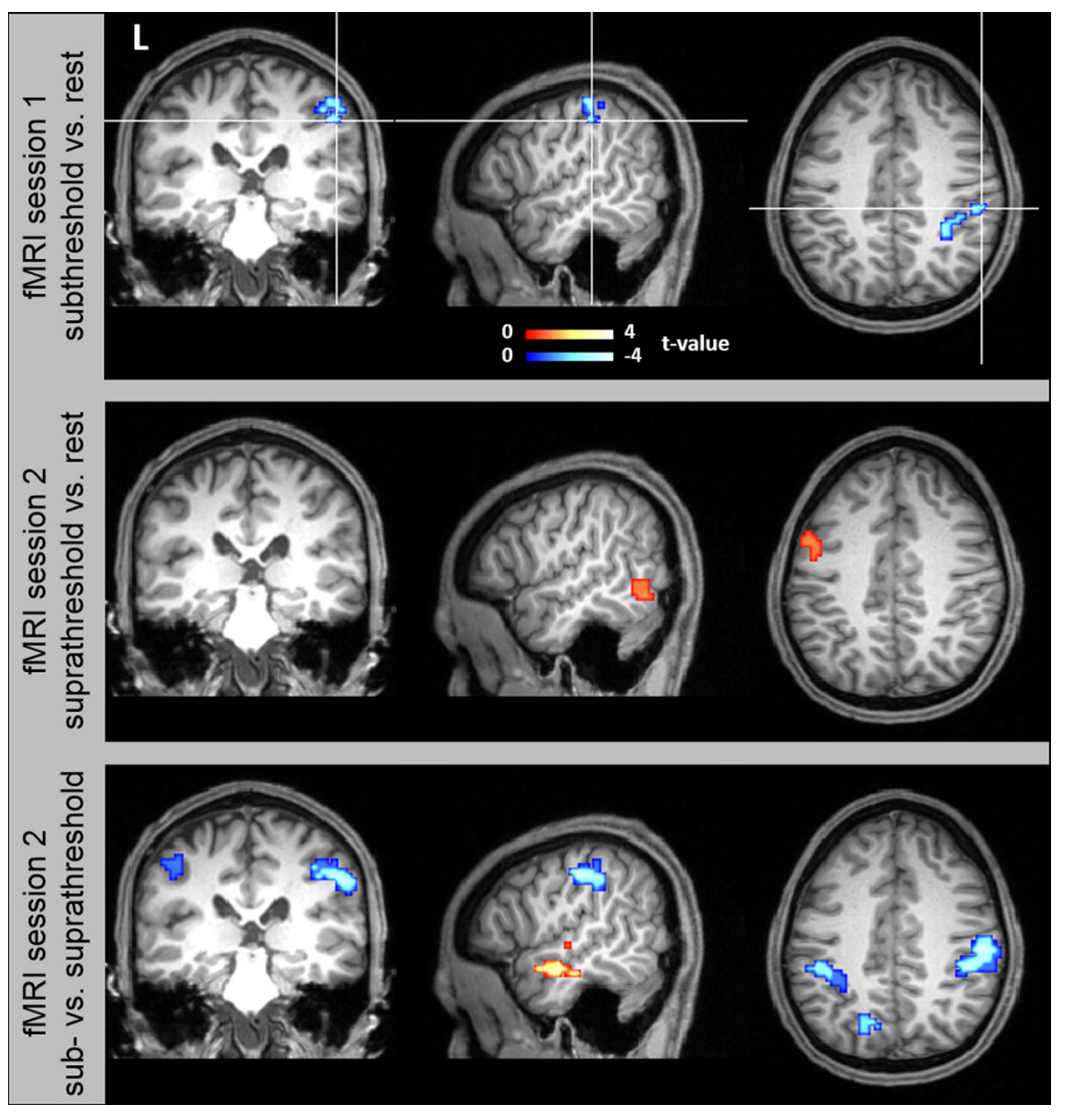

Figure 4. Reduced connectivity of SI during subthreshold stimulation revealed by ECM. Results of a paired $t$ test are shown for contrasting the conditions subthreshold versus rest (top), suprathreshold versus rest (middle), and subthreshold versus suprathreshold (bottom). Warm colors indicate an increase, and cold colors indicate a decrease in centrality (slice coordinates $x=143, y=$ $121, z=67)$. 


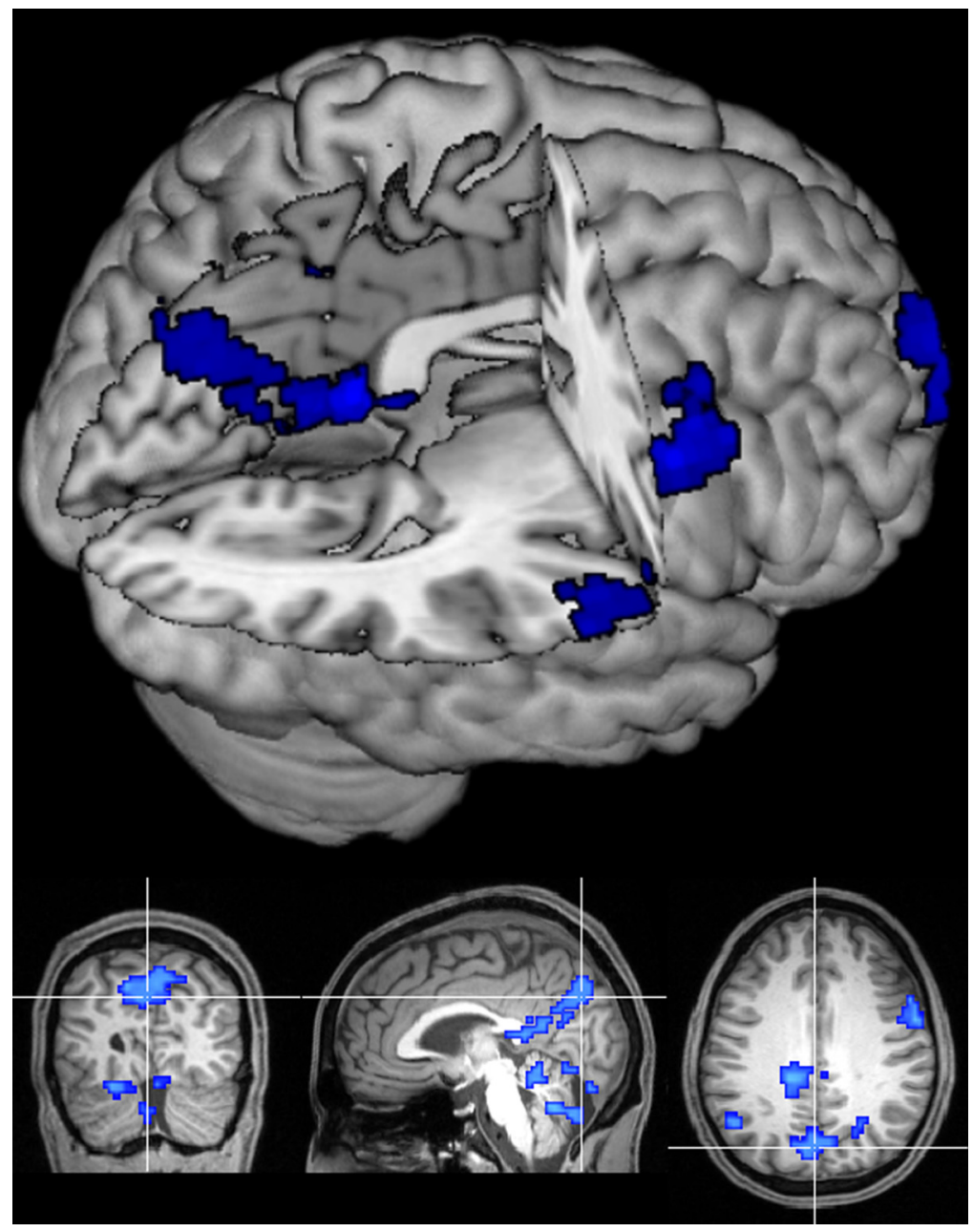

Figure 5. Reduced connectivity of SI to frontoparietal areas revealed by seed-based functional connectivity. The area described by the ECM result (Fig. 4 top, reduced eigenvector centrality during subthreshold stimulation) was used as seed. Blue areas indicate decreased correlation to the seed region, resulting from a paired $t$ test contrasting functional connectivity maps of the subthreshold stimulation block versus rest block (fMRI session 1). Dark blue to light blue means - min to - max (slice coordinates $x=87, y=$ $166, z=76)$.

the opposite, a transient increase in Mu-rhythm strength. Several authors suggested that increases in alpha rhythm indicate a "deactivated" state of the respective cortical area, reflecting predominant inhibitory synaptic processing (Lopes da Silva, 1991; Pfurtscheller et al., 1996; Klimesch et al., 2007; Bollimunta et al., 2008; Jensen et al., 2010, 2012; Buzsáki, 2006). Along with behavioral data supporting this view (Linkenkaer-Hansen et al., 2004; van Dijk et al., 2008; Weisz et al., 2014), the deactivated state is also indexed by the inverse relationship between alpha strength and cortical BOLD signal (Goldman et al., 2002; Laufs et al., 2003; Moosmann et al., 2003; de Munck et al., 2009; Ritter et al., 2009). Also, when inhibiting a response in a motor task, synchronized Mu-rhythm activity and, in a follow-up study, BOLD signal decreases have been observed (Hummel et al., 2002, 2004). The fact that diazepam (a benzodiazepine receptor agonist of the GABA receptor) has been shown to facilitate MEG background rhythms in healthy subjects (Jensen et al., 2005; Hall et al., 2010) also supports a relationship between local alpha activity and inhibitory processing. Thus, the transient $\mathrm{Mu}-$ rhythm increase after subthreshold stimulation may result from a stimulation-induced increase in the magnitude of inhibitory bouts produced by GABAergic interneuronal activity, as proposed previously (Jensen and Mazaheri, 2010).
It seems that after suprathreshold stimulation, a predominant excitatory processing in SI (indexed by an early and strong P1) is followed by local stimulus processing as indexed by the ERD and further ERP components such as the N1, whereas after subthreshold stimulation, a predominant inhibitory processing in SI (indexed by a delayed and weaker P1) prevents further stimulus-specific processing, but triggers a transient elevation of $\mathrm{Mu}$-rhythm as an indicator of a transient downregulation of the system. The latter is consistent with our previous findings in humans that trains of subthreshold stimulation impede the perception of near-threshold stimuli on the same as well as adjacent fingers, accompanied by a focal fMRI deactivation (Blankenburg et al., 2003, Taskin et al., 2008).

Interestingly, an ERD has been described after unperceived near-threshold stimuli (Palva et al., 2005). While we did not investigate the effect of near-threshold stimuli, it seems an intriguing possibility that the bottom-up sweeps of subthreshold and near-threshold stimulation, respectively, are prevented from reaching consciousness at different steps of neural processing. Subthreshold stimuli might be stopped already at the first cortical processing stage in SI, as inhibitory processing outweighs excitatory processing, whereas near-threshold stimuli might be stopped only following more widespread cortical activation but short of conscious perception. Within the nomenclature of Dehaene's global workspace model (Dehaene et al., 2006), near-threshold stimuli might reach a preconscious state, i.e., the necessary conditions for subsequent conscious detection, whereas subthreshold stimuli remain subliminal due to early termination of bottom-up processing.

In general, from our experimental design we cannot differentiate whether the observed effects for subthreshold stimulation are caused by different stimulation strengths or by the absence of perception. As subthreshold stimuli are never consciously detected, a design that contrasts perceived versus unperceived stimuli with the same stimulation strength was not possible. While we speculate that the reduced amplitude of P1 (clearly before any conscious experience) corresponds to the weak stimulation strength, the lack of later ERP components as well as the alpha synchronization may be related to either the difference in stimulation strength and/or the lack of conscious processing. In fact, here we speculate that it is both, i.e., along with the reduced stimulation strength, no further local and widespread processing occurs and hence there is no neural correlate related to conscious processing.

The interpretation of an inhibited access to conscious processing after subthreshold stimulation is further supported by our fMRI findings of reduced connectivity of SI, mainly to areas associated with conscious and higher somatosensory processing. Precuneus and posterior cingulate cortex have not only been de- 
scribed as "higher order motor areas" within a sensorimotor network (Vogt and Vogt, 2003; Margulies et al., 2009; Morecraft et al., 2012), they also seem to be involved in conscious signal processing (Vogt and Laureys, 2005; Cavanna, 2007). Inferior frontal gyrus, operculum, and inferior parietal cortex have been shown to be part of a higher order somatosensory network involved in the processing of tactile stimulation (Dijkerman and de Haan, 2007; Kostopoulos et al., 2007; Spitzer et al., 2014; Weisz et al., 2014). Furthermore, the precuneus-prefrontal connection is associated with conscious perception (Kjaer et al., 2001; Dehaene et al., 2006) and task performance (Utevsky et al., 2014). The decrease in S1 connectivity observed in fMRI after subthreshold stimulation may be closely related to the increased $\mathrm{Mu}$-rhythm, in analogy to the findings of a recent simultaneous EEG fMRI study, which showed that high alpha power in the visual system was associated with decreased local and long-range connectivity of the visual cortex (Scheeringa et al., 2012). A direct link of the two effects is not possible in our data since we did not investigate the same subjects in the EEG and fMRI experiments. However, based on our results it seems reasonable to plan future experiments using simultaneous EEG/fMRI.

In summary, this study provides evidence that for subthreshold stimuli access to conscious perception is prevented as bottom-up processing is terminated at an early stage in SI, likely due to dominant inhibitory processing. A transient predominance of local inhibitory processing would furthermore be consistent with the transient $\mathrm{Mu}$-rhythm increase and the reduced SI connectivity to areas relevant for conscious perception, which can explain the (inhibitory) behavioral effects of trains of subthreshold stimulation on somatosensory perception. We suggest that these processes may serve the suppression of noise and overall the sharpening of somatosensory perception. Notably, this mechanism seems to be generalizable to other sensory systems, as analogous evidence-behaviorally and on alpha rhythm increase (Bareither et al., 2014a, 2014b) — has recently been provided for subliminal visual stimuli.

\section{References}

Allison T, McCarthy G, Wood CC (1992) The relationship between human longlatency somatosensory evoked potentials recorded from the cortical surface and from the scalp. Electroencephalogr Clin Neurophysiol 84: 301-314. CrossRef Medline

Auksztulewicz R, Blankenburg F (2013) Subjective rating of weak tactile stimuli is parametrically encoded in event-related potentials. J Neurosci 33:11878-11887. CrossRef Medline

Bareither I, Villringer A, Busch N (2014a) Decreased visual detection during subliminal stimulation. J Vis 14(12):20. CrossRef Medline

Bareither I, Chaumon M, Bernasconi F, Villringer A, Busch NA (2014b) Invisible visual stimuli elicit increases in alpha-band power. J Neurophysiol 112:1082-1090. CrossRef Medline

Biswal B, Yetkin FZ, Haughton VM, Hyde JS (1995) Functional connectivity in the motor cortex of resting human brain using echo-planar MRI. Magn Reson Med 34:537-541. CrossRef Medline

Blankenburg F, Taskin B, Ruben J, Moosmann M, Ritter P, Curio G, Villringer A (2003) Imperceptible stimuli and sensory processing impediment. Science 299:1864. CrossRef Medline

Bollimunta A, Chen Y, Schroeder CE, Ding M (2008) Neuronal mechanisms of cortical alpha oscillations in awake-behaving macaques. J Neurosci 28:9976-9988. CrossRef Medline

Buzsáki G (2006) Rhythms of the brain. New York: Oxford UP.

Cauller L (1995) Layer I of primary sensory neocortex: where top-down converges upon bottom-up. Behav Brain Res 71:163-170. CrossRef Medline

Cauller LJ, Kulics AT (1991) The neural basis of the behaviorally relevant N1 component of the somatosensory-evoked potential in SI cortex of awake monkeys: evidence that backward cortical projections signal conscious touch sensation. Exp Brain Res 84:607-619. CrossRef Medline
Cavanna AE (2007) The precuneous and consciousness. CNS Spectr 12: 545-552. Medline

Cordes D, Haughton VM, Arfanakis K, Wendt GJ, Turski PA, Moritz CH, Quigley MA, Meyerand ME (2000) Mapping functionally related regions of brain with functional connectivity MR imaging. Am J Neuroradiol 21:1636-1644. Medline

Cordes D, Haughton VM, Arfanakis K, Carew JD, Turski PA, Moritz CH, Quigley MA, Meyerand ME (2001) Frequencies contributing to functional connectivity in the cerebral cortex in "resting-state" data. Am J Neuroradiol 22:1326-1333. Medline

Dehaene S, Changeux JP, Naccache L, Sackur J, Sergent C (2006) Conscious, preconscious, and subliminal processing: a testable taxonomy. Trends Cogn Sci 10:204-211. CrossRef Medline

de Munck JC, Gonçalves SI, Mammoliti R, Heethaar RM, Lopes da Silva FH (2009) Interactions between different EEG frequency bands and their effect on alpha-fMRI correlations. Neuroimage 47:69-76. CrossRef Medline

Dijkerman HC, de Haan EH (2007) Somatosensory processes subserving perception and action. Behav Brain Sci 30:189-201; discussion 201-239. CrossRef Medline

Forman SD, Cohen JD, Fitzgerald M, Eddy WF, Mintun MA, Noll DC (1995) Improved assessment of significant activation in functional magnetic resonance imaging (fMRI): use of a cluster-size threshold. Magn Reson Med 33:636-647. CrossRef Medline

Forschack N, Nierhaus T, Müller MM, Villringer A (2014) Attention modulates cerebral responses to conscious and unconscious tactile events. Poster, OHBM, 20th Annual Meeting, Hamburg.

Fox MD, Raichle ME (2007) Spontaneous fluctuations in brain activity observed with functional magnetic resonance imaging. Nat Rev Neurosci 8:700-711. CrossRef Medline

Fox MD, Snyder AZ, Vincent JL, Corbetta M, Van Essen DC, Raichle ME (2005) The human brain is intrinsically organized into dynamic, anticorrelated functional networks. Proc Natl Acad Sci U S A 102:9673-9678. CrossRef Medline

Fox MD, Snyder AZ, Vincent JL, Raichle ME (2007) Intrinsic fluctuations within cortical systems account for intertrial variability in human behavior. Neuron 56:171-184. CrossRef Medline

Gil Z, Amitai Y (1996) Properties of convergent thalamocortical and intracortical synaptic potentials in single neurons of neocortex. J Neurosci 16:6567-6578. Medline

Goldman RI, Stern JM, Engel J Jr, Cohen MS (2002) Simultaneous EEG and fMRI of the alpha rhythm. Neuroreport 13:2487-2492. CrossRef Medline

Golmayo L, Nuñez A, Zaborszky L (2003) Electrophysiological evidence for the existence of a posterior cortical-prefrontal-basal forebrain circuitry in modulating sensory responses in visual and somatosensory rat cortical areas. Neuroscience 119:597-609. CrossRef Medline

Gusnard DA, Raichle ME (2001) Searching for a baseline: functional imaging and the resting human brain. Nat Rev Neurosci 2:685-694. CrossRef Medline

Hall SD, Barnes GR, Furlong PL, Seri S, Hillebrand A (2010) Neuronal network pharmacodynamics of GABAergic modulation in the human cortex determined using pharmaco-magnetoencephalography. Hum Brain Mapp 31:581-594. CrossRef Medline

Hummel F, Andres F, Altenmüller E, Dichgans J, Gerloff C (2002) Inhibitory control of acquired motor programmes in the human brain. Brain 125:404-420. CrossRef Medline

Hummel F, Saur R, Lasogga S, Plewnia C, Erb M, Wildgruber D, Grodd W, Gerloff C (2004) To act or not to act. Neural correlates of executive control of learned motor behavior. Neuroimage 23:1391-1401. CrossRef Medline

Isaacson JS, Scanziani M (2011) How inhibition shapes cortical activity. Neuron 72:231-243. CrossRef Medline

Jensen O, Mazaheri A (2010) Shaping functional architecture by oscillatory alpha activity: gating by inhibition. Front Hum Neurosci 4:186. CrossRef Medline

Jensen O, Goel P, Kopell N, Pohja M, Hari R, Ermentrout B (2005) On the human sensorimotor-cortex beta rhythm: sources and modeling. Neuroimage 26:347-355. CrossRef Medline

Jensen O, Bonnefond M, VanRullen R (2012) An oscillatory mechanism for prioritizing salient unattended stimuli. Trends Cogn Sci 16:200-206. CrossRef Medline

Kjaer TW, Nowak M, Kjaer KW, Lou AR, Lou HC (2001) Precuneus- 
prefrontal activity during awareness of visual verbal stimuli. Conscious Cogn 10:356-365. CrossRef Medline

Klimesch W, Sauseng P, Hanslmayr S (2007) EEG alpha oscillations: the inhibition-timing hypothesis. Brain Res Rev 53:63-88. CrossRef Medline

Knight RT, Staines WR, Swick D, Chao LL (1999) Prefrontal cortex regulates inhibition and excitation in distributed neural networks. Acta Psychol 101:159-178. CrossRef Medline

Kostopoulos P, Albanese MC, Petrides M (2007) Ventrolateral prefrontal cortex and tactile memory disambiguation in the human brain. Proc Natl Acad Sci U S A 104:10223-10228. CrossRef Medline

Laufs H, Kleinschmidt A, Beyerle A, Eger E, Salek-Haddadi A, Preibisch C, Krakow K (2003) EEG-correlated fMRI of human alpha activity. Neuroimage 19:1463-1476. CrossRef Medline

Li Y, Ma Z, Lu W, Li Y (2006) Automatic removal of the eye blink artifact from EEG using an ICA-based template matching approach. Physiol Meas 27:425-436. CrossRef Medline

Libet B, Alberts WW, Wright EW Jr, Feinstein B (1967) Responses of human somatosensory cortex to stimuli below threshold for conscious sensation. Science 158:1597-1600. CrossRef Medline

Linkenkaer-Hansen K, Nikulin VV, Palva S, Ilmoniemi RJ, Palva JM (2004) Prestimulus oscillations enhance psychophysical performance in humans. J Neurosci 24:10186-10190. CrossRef Medline

Lohmann G, Müller K, Bosch V, Mentzel H, Hessler S, Chen L, Zysset S, von Cramon DY (2001) LIPSIA-a new software system for the evaluation of functional magnetic resonance images of the human brain. Comput Med Imaging Graph 25:449-457. CrossRef Medline

Lohmann G, Margulies DS, Horstmann A, Pleger B, Lepsien J, Goldhahn D, Schloegl H, Stumvoll M, Villringer A, Turner R (2010) Eigenvector centrality mapping for analyzing connectivity patterns in fMRI data of the human brain. PLoS One 5:e10232. CrossRef Medline

Lopes da Silva F (1991) Neural mechanisms underlying brain waves: from neural membranes to networks. Electroencephalogr Clin Neurophysiol 79:81-93. CrossRef Medline

Margulies DS, Vincent JL, Kelly C, Lohmann G, Uddin LQ, Biswal BB, Villringer A, Castellanos FX, Milham MP, Petrides M (2009) Precuneus shares intrinsic functional architecture in humans and monkeys. Proc Natl Acad Sci U S A 106:20069-20074. CrossRef Medline

Margulies DS, Böttger J, Long X, Lv Y, Kelly C, Schäfer A, Goldhahn D, Abbushi A, Milham MP, Lohmann G, Villringer A (2010) Resting developments: a review of fMRI post-processing methodologies for spontaneous brain activity. MAGMA 23:289-307. CrossRef Medline

Miller KD, Pinto DJ, Simons DJ (2001) Processing in layer 4 of the neocortical circuit: new insights from visual and somatosensory cortex. Curr Opin Neurobiol 11:488-497. CrossRef Medline

Moosmann M, Ritter P, Krastel I, Brink A, Thees S, Blankenburg F, Taskin B, Obrig H, Villringer A (2003) Correlates of alpha rhythm in functional magnetic resonance imaging and near infrared spectroscopy. Neuroimage 20:145-158. CrossRef Medline

Morecraft RJ, Stilwell-Morecraft KS, Cipolloni PB, Ge J, McNeal DW, Pandya DN (2012) Cytoarchitecture and cortical connections of the anterior cingulate and adjacent somatomotor fields in the rhesus monkey. Brain Res Bull 87:457-497. CrossRef Medline

Neuper C, Pfurtscheller G (2001) Event-related dynamics of cortical rhythms: frequency-specific features and functional correlates. Int J Psychophysiol 43:41-58. CrossRef Medline

Nierhaus T, Margulies DS, Long X, Villringer A (2012) fMRI for the assessment of functional connectivity. In: Neuroimaging methods (Bright P, ed). Rijeka: In Tech.

Oldfield RC (1971) The assessment and analysis of handedness: the Edinburgh inventory. Neuropsychologia 9:97-113. CrossRef Medline

Palva S, Linkenkaer-Hansen K, Näätänen R, Palva JM (2005) Early neural correlates of conscious somatosensory perception. J Neurosci 25:52485258. CrossRef Medline

Pfurtscheller G, Stancák A Jr, Neuper C (1996) Event-related synchronization (ERS) in the alpha band - an electrophysiological correlate of cortical idling: a review. Int J Psychophysiol 24:39-46. CrossRef Medline
Ploner M, Gross J, Timmermann L, Pollok B, Schnitzler A (2006) Pain suppresses spontaneous brain rhythms. Cereb Cortex 16:537-540. CrossRef Medline

Poline JB, Worsley KJ, Evans AC, Friston KJ (1997) Combining spatial extent and peak intensity to test for activations in functional imaging. Neuroimage 5:83-96. CrossRef Medline

Ray PG, Meador KJ, Smith JR, Wheless JW, Sittenfeld M, Clifton GL (1999) Physiology of perception: cortical stimulation and recording in humans. Neurology 52:1044-1049. CrossRef Medline

Ritter P, Moosmann M, Villringer A (2009) Rolandic alpha and beta EEG rhythms' strengths are inversely related to fMRI-BOLD signal in primary somatosensory and motor cortex. Hum Brain Mapp 30:1168-1187. CrossRef Medline

Scheeringa R, Petersson KM, Kleinschmidt A, Jensen O, Bastiaansen MC (2012) EEG alpha power modulation of fMRI resting-state connectivity. Brain Connect 2:254-264. CrossRef Medline

Schubert R, Blankenburg F, Lemm S, Villringer A, Curio G (2006) Now you feel it-now you don't: ERP correlates of somatosensory awareness. Psychophysiology 43:31-40. CrossRef Medline

Spitzer B, Goltz D, Wacker E, Auksztulewicz R, Blankenburg F (2014) Maintenance and manipulation of somatosensory information in ventrolateral prefrontal cortex. Hum Brain Mapp 35:2412-2423. CrossRef Medline

Srisa-an P, Lei L, Tarkka IM (1996) Middle latency somatosensory evoked potentials: noninvasive source analysis. J Clin Neurophysiol 13:156-163. CrossRef Medline

Staines WR, Graham SJ, Black SE, McIlroy WE (2002) Task-relevant modulation of contralateral and ipsilateral primary somatosensory cortex and the role of a prefrontal-cortical sensory gating system. Neuroimage 15: 190-199. CrossRef Medline

Stancák A (2006) Cortical oscillatory changes occurring during somatosensory and thermal stimulation. Prog Brain Res 159:237-252. CrossRef Medline

Swadlow HA (2003) Fast-spike interneurons and feedforward inhibition in awake sensory neocortex. Cereb Cortex 13:25-32. CrossRef Medline

Taskin B, Holtze S, Krause T, Villringer A (2008) Inhibitory impact of subliminal electrical finger stimulation on SI representation and perceptual sensitivity of an adjacent finger. Neuroimage 39:1307-1313. CrossRef Medline

Utevsky AV, Smith DV, Huettel SA (2014) Precuneus is a functional core of the default-mode network. J Neurosci 34:932-940. CrossRef Medline

van Dijk H, Schoffelen JM, Oostenveld R, Jensen O (2008) Prestimulus oscillatory activity in the alpha band predicts visual discrimination ability. J Neurosci 28:1816-1823. CrossRef Medline

Vogt BA, Laureys S (2005) Posterior cingulate, precuneal and retrosplenial cortices: cytology and components of the neural network correlates of consciousness. Prog Brain Res 150:205-217. CrossRef Medline

Vogt BA, Vogt L (2003) Cytology of human dorsal midcingulate and supplementary motor cortices. J Chem Neuroanat 26:301-309. CrossRef Medline

Weisz N, Wühle A, Monittola G, Demarchi G, Frey J, Popov T, Braun C (2014) Prestimulus oscillatory power and connectivity patterns predispose conscious somatosensory perception. Proc Natl Acad Sci U S A 111: E417-E425. CrossRef Medline

Whitehorn D, Towe AL (1968) Postsynaptic potential patterns evoked upon cells in sensorimotor cortex of cat by stimulation at the periphery. Exp Neurol 22:222-224. CrossRef Medline

Wikström H, Huttunen J, Korvenoja A, Virtanen J, Salonen O, Aronen H, Ilmoniemi RJ (1996) Effects of interstimulus interval on somatosensory evoked magnetic fields (SEFs): a hypothesis concerning SEF generation at the primary sensorimotor cortex. Electroencephalogr Clin Neurophysiol 100:479-487. CrossRef Medline

Zhang Y, Ding M (2010) Detection of a weak somatosensory stimulus: role of the prestimulus mu rhythm and its top-down modulation. J Cogn Neurosci 22:307-322. CrossRef Medline 